\title{
„Sein Unglück ausatmen können“. Hilfe für die Helfenden
}

\author{
Christoph Seidl
}

In der Diözese Regensburg gibt es die Stelle „Seelsorge für Berufe im Gesundheits- und Sozialwesen“, die aus den früheren Bereichen „Seelsorge für Pflegeberufe“ und „Seelsorge für Beratungsdienste“ entstanden ist. Dieser Fachbereich ist gekoppelt mit der Koordination der Krankenhaus- und Hospizseelsorge im Bistum. Aus dieser Perspektive ist es besonders interessant zu reflektieren, was diese helfenden Berufe in der Krisenzeit besonders belastet und was ihnen selbst seelsorglich guttun kann.

Helfende Berufe leisten auch in schwierigen Zeiten das, wozu sie ausgebildet wurden und wozu sie angetreten sind: nämlich anderen in ihrer Not beizustehen, sie zu unterstützen, sie zu entlasten. Aber in einer Pandemie können die eigenen Kräfte rasch an die Grenzen gelangen. Dass Pflegende und Beratende systemrelevant sind, steht außer Frage. Aber ist es Seelsorge? Die Krise macht deutlich, dass sie eminent dazu beiträgt, denen „in vorderster Linie“ den Rücken zu stärken, indem sie „Atemraum“ schafft.

\section{Die Krise - Normalfall für helfende Berufe oder mehr?}

In der intensiven Corona-Phase gab es unterschiedliche Situationen bei den Pflegenden: Die einen waren durch die unmittelbare Arbeit mit COVID-19-PatientInnen bis an ihre Grenzen beschäftigt, andere wurden heimgeschickt, weil das „Alltagsgeschäft“ in den Kliniken einbrach. Unterschiedliches Arbeitsaufkommen wurde auch von den Personen in den Beratungsdiensten erlebt. Insofern gibt es auch verschiedene Perspektiven auf das Krisengeschehen.

\section{1 „Ich tu das, was ich immer tu! ““}

Eine Krankenpflegerin hat es mit großer Ausgeglichenheit formuliert, was ich mehrfach bestätigt gefunden habe: „Ich tu das, was ich immer tu!" Als sie berichtet, dass sie auch mit ihrer verminderten Herzleistung in der intensi- 
ven Coronaphase ihren Dienst im Krankenhaus ausübt, wundere ich mich sehr. Aber sie sagt:

"Wieso sollte ich jetzt nicht arbeiten? Wir haben in der Ausbildung gelernt: Begegne jedem Patienten so, als ob er infektiös sein könnte! Das mache ich immer - und das mache ich auch jetzt, denn jetzt werde ich gebraucht, so wie ich immer gebraucht werde!"

Die 53-Jährige schmälert nicht die Gefährlichkeit der Pandemie und hält die Schutzmaßnahmen sowie die Beschränkungen im Alltag für völlig korrekt. Dennoch entdecke ich bei ihr und nicht wenigen Pflegenden ein großes Maß an nüchterner Betrachtungsweise.

Das Gesagte gilt auch für Beratende. Sie haben ja immer mit Menschen in persönlichen Krisensituationen zu tun. Sie bieten immer einen Raum an, in dem Betroffene „ihr Unglück ausatmen“ können. Sie versuchen nach allen Regeln der Kunst, Perspektivenwechsel zu ermöglichen und Sortierhilfen anzubieten, um im Dickicht der Sorgen einen gangbaren Weg zu entdecken.

Derart eingeübte Verhaltensweisen sind in Krisenzeiten gute Ressourcen. Und doch ist die Pandemie eine Krise von außergewöhnlichem Ausmaß.

\subsection{Pandemie: Die Krise betrifft alle und alles}

Es geht in dieser Situation nicht nur um die Krise eines Patienten oder Klienten, es ist eine Krise, in die jeder und jede miteinbezogen ist. Pflegende haben Sorge um die eigene Gesundheit und die ihrer Angehörigen und Freunde. Beratende wissen vom Verlauf und vom Umfang der Krise bisweilen ebenso wenig wie die Ratsuchenden. Freilich haben Beratende durch Ausbildung und Berufserfahrung bessere Voraussetzungen, um mit derartigen Krisen umzugehen, aber Ängste machen sich auch unter „Profis" breit. Die Krise nimmt den Helfenden mit hinein wie in einen Strudel. Vielleicht könnte man sie mit einem Phänomen vergleichen, das von der HIV-Infektion bekannt ist: Die Krise ist auch im Helfer vorhanden, nicht nur die Angst vor der Krankheit, sondern sogar vor dem zu betreuenden Menschen. Eigentlich widerspricht das dem Grundauftrag des Helfers, der seinem Gegenüber ja als Außenstehender begegnen sollte. Sein eigenes „inneres Team“ ist erschüttert, denn es fehlt der „Schlichter“ für die eigenen inneren Konflikte. Die allgegenwärtige Angst kann Vertrauen stören, das bisher nicht in Frage gestellt war. Eine Beratungsperson sagt mit Hinblick auf die unsichere Ausstattung mit Mund-Nasen-Schutzmasken: 
„Wenn mich mein Dienstgeber nicht schützen kann, gehe ich nicht mehr zur Arbeit." Die Expertise der „Fachperson“ fällt teilweise weg, da diese selbst vom Problem betroffen ist. Nicht zuletzt wird die Grenze zwischen Dienst und Privatleben fließend: Die Maske, die Pflegende in der Klinik tragen, brauchen sie nun auch beim Einkaufen und beim Besuch der Eltern wenn sie überhaupt zu besuchen sind. Die Krisen im Beruf und zuhause vermischen sich. Es entsteht eine seltsame Gemengelage der „Erholungslosigkeit“.

\title{
1.3 Ausatmen und einatmen-GEIST-liche Begleitung für Helfende
}

Wie kann Seelsorge unter solchen außergewöhnlichen Bedingungen helfenden Berufen zur Seite stehen? Das Gedicht Aufhebung ${ }^{1}$ von Erich Fried (1921-1988) bringt in lyrischer Weise auf den Punkt, was Seelsorge für helfende Berufe gerade in Krisenzeiten bedeuten könnte:

\author{
Aufhebung \\ Sein Unglück \\ ausatmen können
}

tief ausatmen so dass man wieder einatmen kann

Und vielleicht auch sein Unglück sagen können in Worten in wirklichen Worten die zusammenhängen

und Sinn haben und die man selbst noch verstehen kann und die vielleicht sogar irgendwer sonst versteht oder verstehen könnte

Und weinen können das wäre schon fast wieder Glück

1 Siehe Fried, Aufhebung. 
Das Wortpaar ausatmen - einatmen aus Erich Frieds Gedicht Aufhebung formuliert sehr schön, was Seelsorge anbieten kann: einen Raum, um atmen zu können. Das Buch Genesis beschreibt auf den ersten Seiten der Bibel das Entscheidende, das den Menschen zum Menschen macht, als den göttlichen Lebensatem, der dem Adam, dem Wesen aus Ackererde, eingehaucht wird. In allen alten Sprachen ist das Wort für Atem und für Geist dasselbe: hebräisch ruach, griechisch pneuma, lateinisch spiritus. Atman bedeutet in der indischen Philosophie so viel wie Seele, bezeichnet also das Göttliche selbst. Geistliche Begleitung ist demnach die Unterstützung von Menschen, die in Krisenzeiten besonders herausgefordert sind, sich auf die wesentlichen menschlichen Existenzbedingungen zu konzentrieren. Seelsorge hat den Auftrag, Räume zur Verfügung zu stellen, in denen ihnen der göttliche Lebensatem neu bewusst wird, der sie antreibt, motiviert, den rechten Weg erkennen lässt, so dass ihnen die sprichwörtliche Luft nicht ausgeht.

Was es bewirkt, diesen Lebensatem neu zu entdecken, beschreibt ein Vers im zweiten Brief des Apostels Paulus an seinen Mitarbeiter Timotheus. Dort heißt es: „Gott hat uns nicht einen Geist der Verzagtheit gegeben, sondern den Geist der Kraft, der Liebe und der Besonnenheit." (2 Tim 1,6f) Seelsorgliche Begleitung bietet einen Raum der Be-SINN-ung, um die möglicherweise verlorene Besonnenheit wiederzugewinnen. Das Thema Sinnfindung ist in unübersichtlichen Zeiten besonders brisant, dazu ist ein Gegenüber hilfreich, mit dem Sinnperspektiven erörtert werden können. Aus der Notfallseelsorge ist der Grundsatz geläufig, dass in Zeiten starker Verunsicherung zunächst einmal Ruhe und Struktur notwendig sind, um in dem heillosen Durcheinander wieder sinnvolle nächste Schritte zu erkennen. Auf diese Weise wird es möglich, eigene Kräfte zu reaktivieren, die manchmal schwer aufzubringende Liebe zu den anvertrauten Personen zumindest als „Pro-Existenz“ (Einsatz für) zu begreifen und schließlich in der Aufregung der bedrängenden Situation erst dreimal tief durchzuatmen, bevor möglicherweise nicht genügend durchdachte Entscheidungen getroffen werden.

\section{2. „Dein Ort ist, wo Augen dich ansehen" (H. Domin) - Plädoyer für eine Aufwertung durch mehr Ansehen}

Entscheidend für eine gute seelsorgliche, lebensförderliche Begleitung ist es, vor jeglicher Intervention oder gar einem Rat zu sehen, was ist, und zwar nicht nur die Situation oder den „Fall“, sondern in erster Linie den Menschen, der Unterstützung benötigt. In einem Gedicht der deutschen Lyri- 
kerin Hilde Domin (1909-2006) kommt die existenzielle Bedeutung des Ansehens sehr stark zum Ausdruck. Es trägt den Titel Es gibt dich²:

Dein Ort ist

wo Augen dich ansehen.

Wo sich Augen treffen

entstehst du.

Von einem Ruf gehalten, immer die gleiche Stimme, es scheint nur eine zu geben mit der alle rufen.

Du fielest, aber du fällst nicht. Augen fangen dich auf.

Es gibt dich weil Augen dich wollen, dich ansehen und sagen dass es dich gibt.

Einander in die Augen zu sehen und im Blick zu haben, ist eine Grundlage menschlichen Zusammenlebens. Das gilt für die Begegnung von Helfenden und Hilfesuchenden, aber ebenso für die Zusammenarbeit in einem Team. Nicht von ungefähr gibt es den schönen sprachlichen Zusammenhang zwischen jemanden ansehen und jemandem Ansehen geben. Blickkontakt hat mit Würde zu tun, biblisch gesprochen mit Ebenbildlichkeit. Die Bibel betont wiederholt, wie wichtig es ist, dass Gott die Menschen im Blick hat: "Verbirgst du dein Gesicht, sind sie (die Geschöpfe) verstört." (Ps 104,29) Im Ansehengeben liegt auch Segen: „Der HERR wende sein Angesicht dir zu und schenke dir Frieden. " (Dtn 6,26)

Gerade unter dem fehlenden Sehen und Gesehenwerden leiden während der strengen Distanzregelungen auch viele Angehörige der helfenden Berufe.

2 Domin, Es gibt dich, 56. 


\subsection{Herausforderungen in der Pflege}

Pflegende ziehen viel Kraft aus dem Wissen, Menschen in bedrohlichen Situationen helfen zu können. Dazu gehört wesentlich das Feedback von Patienten und Angehörigen, aber auch vom Team bzw. von der eigenen Einrichtung. Es geht um das Sehen der Not und das Gesehenwerden als „Nothelfer“. Pflegende, die verstärkt mit COVID-19-Patienten zu tun hatten, litten darunter, dass dieses Verhältnis von Einsatz und Sinnerfüllung in Schieflage geraten ist. Dazu trug zum einen die Zunahme an Todesfällen bei. Freilich sind es Pflegepersonen gewohnt, dass auch Menschen sterben, aber die geballte Erfahrung von Todesfällen macht auch Fachkräften zu schaffen.

Ein weiteres großes Problem für Pflegende in Einrichtungen der Altenhilfe war das Besuchsverbot. Pflegende wurden auf diese Weise über längere Zeit zu den einzigen Bezugspersonen. Das kann neben der gewöhnlichen Betreuungsarbeit schnell zu einer Überforderung werden. Besonders dramatisch haben es Pflegende erlebt, wenn es Bewohnern mit einer Demenzerkrankung nicht zu erklären war, warum die Angehörigen derzeit nicht kommen dürfen.

Schließlich treten in Krisenzeiten Schwachpunkte der Organisation bzw. des alltäglichen Erlebens besonders zutage. Fehlt sonst schon das Gefühl der Anerkennung und des Ansehens in der eigenen Arbeit, so ist dieser Mangel unter Extrembedingungen besonders spürbar. Dramatischer waren die Kompensation von krankheitsbedingten Ausfällen und die sonst schon schwierige Dienstplangestaltung. Der eigene Gesundheitsschutz, der an sich immer wichtig ist, wurde plötzlich auf Schritt und Tritt thematisiert. Ausgleich und Ermutigung im Team und im Privatleben wichen einer permanenten Ungewissheit, wie die Pandemie sich entwickeln würde, ob das Krankheitsaufkommen noch zu bewältigen wäre oder ob - wie in anderen Ländern - das Gesundheitssystem völlig kollabieren könnte.

\subsection{Herausforderungen für die Beratungsdienste}

Das größte Problem für Beratungsdienste in dieser Zeit war der Umstand, dass sämtliche Face-to-Face-Begegnungen auf Telefonkontakte umgestellt werden mussten. Video-Kontakte waren in vielen kirchlichen Beratungsstellen zum Teil aus technischen, zum Teil aus Datenschutzgründen nicht oder nur schwer möglich. Selbst wenn Video-Beratung angeboten werden konnte, wurde dennoch die schlechtere Qualität der Zweierbegegnung im Vergleich zum Echtkontakt beklagt. 
Aus der Ehe-, Familien- und Lebensberatung war die Klage zu vernehmen, dass es schier unmöglich sei, mit Paaren gleichzeitig per Telefon zu kommunizieren - das wäre aber grundlegend für Beratungsprozesse. Denn es ist von Bedeutung, wie ein Partner reagiert, wenn er in Anwesenheit eines Dritten vom anderen Dinge hört, die er eventuell so noch nicht gehört hat oder bisher nicht hören wollte.

Auch der Blickkontakt im Kollegenkreis der Beratungsstelle war streckenweise nicht mehr möglich. Beim Aufenthalt im Homeoffice konnte man sich in Pausen nicht kurz zwischen Tür und Angel austauschen. Dieser Austausch ist jedoch ein wichtiges Moment von Selbstsorge. Es tut einfach gut, gemeinsam durchatmen zu können, auch mal kurz übers Wetter, das geplante Konzert oder auch ein persönliches Familienthema sprechen zu können. Eine Beraterin sagt:

„Ich bin meinen eigenen Gedanken so ausgeliefert, wenn da kein Korrektiv da ist (nicht mal am Stammtisch), da wird man sonst nämlich schnell wieder eingenordet. Aber wenn ich dauernd mit mir und meinen Klienten alleine bin, dann geht das an die Substanz."

Aus der Beratungsstelle für seelische Gesundheit wurde folgende Beobachtung geschildert:

„Mittlerweile machen mir die Blüten Angst, die das Ganze treibt. Mit den ,normalen'Zwangs- und Angststörungen können wir ja umgehen. Aber momentan verstärken sich diese Auffälligkeiten und es kommt zu absolut absurden Reaktionen. Verschwörungstheorien sind das eine, aber manche ticken so aus, dass sich auch unser Empathievermögen in Grenzen hält."

Unterschiedliche Beratungsdienste berichten übereinstimmend vom Überlagern der an sich schon komplexen Problemlagen mit den wirtschaftlichen Sorgen und den vielfachen Ängsten, die die Kontaktverbote auslösen können. Manche Klienten, die unter Normalbedingungen schon schwer am Leben zu tragen haben, sehen sich durch zusätzliche Regeln um ihre Freiheitsrechte betrogen und reagieren bisweilen unverhältnismäßig heftig.

\subsection{DANKE allein genügt noch nicht-Auf-Wertung ist notwendig}

„Dein Ort ist, wo Augen dich ansehen!" Das gilt für die Patienten und Klienten ebenso wie für die Helfenden. Aber genau an dieser Stelle macht die Corona-Krise etwas Trauriges deutlich: die helfenden Berufe leiden unter 
normalen Bedingungen schon unter einem beträchtlichen Mangel an Ansehen. Wenn nun auch noch das - ganz wörtlich verstandene - Ansehen der eigenen Klientel fehlt, dann bricht tatsächlich ein Kernstück des Selbstwertgefühls weg.

Paradoxerweise hilft es auch nicht, dass nun von verschiedenen Seiten die Pflege und die Kriseninterventionsdienste plötzlich als systemrelevant bezeichnet werden und z. B. eine Sonderprämie von 500 Euro für eine Pflegeperson in Aussicht gestellt wird. Gleichzeitig wird natürlich auch gefragt, woher das Geld dazu kommen soll (bei Prämien für Autokäufe wird diese Frage seltsamerweise nicht gestellt). Vor kurzem wurde noch diskutiert, dass 30 Prozent aller Kliniken in Deutschland wegzurationalisieren wären, plötzlich werden zu Rehaeinrichtungen umfunktionierte alte Krankenhäuser wieder zu Corona-Zentren umgerüstet. In dieser Zeit gab es mehrere Vorstöße von Interessensverbänden, die Pflege und andere helfende Berufe über die Krise hinaus wieder nachhaltig aufzuwerten. Ebenso gerieten die Einrichtungen der Altenhilfe massiv in den Fokus, die öffentliche Aufmerksamkeit war verstärkt auf menschenwürdige Zustände ausgerichtet. Man darf gespannt bleiben, wer sich nach der Krise noch an all diese Themen erinnern wird. Es wird deutlich, dass Krankenhäuser sowie Alten- und Pflegeheime nicht gut Wirtschaftsunternehmen sein können, die sich selbst finanzieren bzw. Gewinn erzielen müssen. Es geht um die Frage, welches Ansehen den Menschen zukommen soll, die diese Systeme tragen - nicht einmalig, sondern dauerhaft. An der Frage, mit welchen Augen kranke und pflegebedürftige Menschen gesehen werden, entscheidet sich auch die Ernsthaftigkeit, mit der die Diskussion um die Menschenwürde verbunden ist. Sie beginnt nicht erst bei der Verfügbarkeit eines Beatmungsgerätes für einen COVID-19-Patienten, sondern bei der Wertschätzung von Menschen in Gesundheits- und Sozialberufen.

\section{3. „Wo aber Gefahr ist, wächst das Rettende auch"3 (F. Hölderlin) - Rückenwind für die kategoriale Seelsorge}

Die Zeit der starken Beschränkungen des öffentlichen Lebens hat der Seelsorge und den Sorgebeziehungen viel abverlangt. Aber es ist zugleich et-

3 Das Zitat stammt aus der 1803 vollendeten Hymne Patmos von Friedrich Hölderlin. Das Gedicht ist nach der griechischen Insel Patmos benannt, die als Entstehungsort der biblischen Offenbarung des Johannes gilt. Sie ist eine Trost- und Hoffnungsschrift für Christen in der Verfolgungssituation des Römischen Reiches und kennzeichnet zugleich die apokalyptische Krisensituation. 
was sichtbar geworden, was den künftigen Stellenwert der Seelsorge sogar stärken könnte. Zum einen könnte der Blick von außen auf Seelsorge wertschätzender werden (Stichwort „systemrelevant“), zum anderen könnte innerkirchlich die Bedeutung der kategorialen Seelsorge verstärkt wahrgenommen werden, hat sich doch gezeigt, dass unter diesen extremen Bedingungen die Seelsorge im jeweiligen System am ehesten handlungsfähig geblieben ist.

\subsection{Nachgehende Seelsorge: In Beziehung kommen - in Beziehung bleiben}

Papst Franziskus hat mit seiner Aufforderung zu „einem beständigen Aufbruch zu den Peripherien des eigenen Territoriums oder zu den neuen soziokulturellen Umfeldern" ${ }^{4}$ etwas in Erinnerung gerufen, was seit dem II. Vatikanum immer wieder thematisiert wird und seit biblischen Zeiten im Grundbuch der Christenheit eingetragen ist: Kirche muss unterwegs zu den Menschen sein, sie darf nicht warten, bis und ob die Menschen zu ihr kommen.

„Kirche in der Welt von heute" wird besonders sichtbar, wenn sie als Seelsorge in bestimmten Bereichen und Systemen „vor Ort" ist. Gerade in den Bereichen Krankenhaus- und Hospizseelsorge hat sich in den letzten Jahrzehnten eine hervorragende Art der Zusammenarbeit ergeben. Kirchliche Seelsorge hat es verstanden, sich als willkommener "Gast" auf einem anderen hoheitlichen Gebiet zu verstehen. Sie hat gelernt, als Teil eines interdisziplinären Systems anderen Professionen wie Medizin, Pflege und sozialen Diensten auf Augenhöhe, also als Partner, zu begegnen. Dazu leistete eine Einladung „von außen“ einen wesentlichen Beitrag. Die englische Krankenschwester, Sozialarbeiterin und Ärztin Cicely Saunders (19182005) betonte, dass es die Aufgabe von Begleitung am Lebensende ist, alles zu tun, damit schwerkranke Menschen ,nicht nur in Frieden sterben, sondern auch bis zuletzt leben können" ${ }^{\text {. }}$. Die Weltgesundheitsorganisation WHO hat im Anschluss daran im Jahr 2002 definiert, die Aufgaben von Palliative Care seien das „Vorbeugen und Lindern von Leiden durch frühzeitiges Erkennen, Einschätzen und Behandeln von Schmerzen sowie anderer belastender Beschwerden körperlicher, psychosozialer und spiritueller Art"6. Es gehört zum Selbstverständnis von Palliative Care, dass die spirituelle Sorge, in unseren Brei-

4 Papst Franziskus, Apostolisches Schreiben Evangelii Gaudium, 30.

5 Saunders, zitiert nach Palliamo.

6 Weltgesundheitsorganisation (WHO), Definition of Palliative Care 2002. 
ten nicht selten angeboten durch die kirchliche Seelsorge, unverzichtbar zum System gehört. Wo immer solches Zusammenwirken gelingt - auf Palliativstationen und in Hospizen an der Tagesordnung, in Krankenhäusern vielerorts gut eingespielt -, wird sichtbar, wie sehr Seelsorge für alle Beteiligten, sowohl für die PatientInnen als auch für die anderen Berufsgruppen, eine Bereicherung darstellen und einen wesentlichen Beitrag für die Bewältigung von Krisen leisten kann.

Seelsorge beginnt nicht mit liturgischem Handeln, sondern im Mitgehen von Lebenswegen und im Mitaushalten von Leidenssituationen, vielleicht beim Halten der Hand am Krankenbett. In der Krise hat sich gezeigt, dass Seelsorge im System Krankenhaus sehr wohl gefragt war. Für Gemeinde-Seelsorge war es so gut wie nicht möglich, Zugang in die Einrichtung zu bekommen. Kirchenleitungen und Personalabteilungen sollten diese Chance der kategorialen Seelsorge auch mit Blick auf künftige Pastoralplanungen nicht leichtfertig verspielen.

\subsection{Lebensbejahende Seelsorge: Glaube - Hoffnung - Liebe}

Weniger öffentlichkeitswirksam als Liturgie und Ethik ist die ganz grundlegende Unterstützung von Menschen in der Bewältigung der Krise - ich nenne es den Dienst der Lebensbejahung. Und ich wähle für einen Blick darauf die biblische Trias Glaube, Hoffnung und Liebe aus dem Korintherbrief des Apostels Paulus (1 Kor 13).

\subsubsection{Glaube: „Vertikale Resonanz“ (H. Rosa)}

Wie oben erwähnt, unterstreicht die WHO die spirituelle Dimension von menschlichem Leid. Freilich ist spirituell hier ein sehr weitgefasster Begriff für das Bedürfnis nach Sinn und Orientierung. Aber immerhin können sich hier Medizin und Theologie hervorragend treffen. Dabei steht freilich nicht das Glaubensbekenntnis im Vordergrund, sondern vielmehr die Auseinandersetzung um Sinn und Bedeutung der eigenen Lebenslage.

Der Soziologe Hartmut Rosa schreibt in seinem Buch Resonanz $z^{7}$ über die Dimensionen eines gelingenden Lebens. Ohne Zweifel gehört für ihn neben der horizontalen (Begegnung) und der diagonalen (Interessen, Be-

7 Siehe Rosa, Resonanz. 
schäftigung) die „vertikale Resonanz" ${ }^{\text {, }}$, die Verankerung in einem Sinn oder einer Idee, ganz wesentlich zum Leben. Lebens- und Leiddeutung, wie sie der Glaube anbietet, ist also durchaus ein wesentlicher Beitrag zur Krisenbewältigung. Hartmut Rosa beklagt in einem Interview im Deutschlandfunk ${ }^{9}$, Kirchen und Religionsvertreter hätten in der Krise eine gewisse Mutlosigkeit gezeigt. Sie hätten eine sehr defensive Einstellung, ein Gefühl, dass die Gesellschaft nichts von ihnen wissen wolle. Dabei wäre es an der Zeit, in der Gesellschaft eine religiöse Stimme zu hören, näherhin sogar eine christliche. Das Virus habe der Bevölkerung wieder ins Bewusstsein gebracht, dass bestimmte Dinge auch in einer modernen Kultur unverfügbar und nicht beeinflussbar sind. Von daher hat Seelsorge aus der Perspektive des Glaubens einen sehr wichtigen Beitrag zu leisten.

\subsubsection{Hoffnung: „... trotzdem Ja zum Leben sagen“(V. E. Frankl)}

Von Hoffnung wird in Krisenzeiten viel geredet, meistens geht es um die Hoffnung, dass das Schwere bald vorübergeht und alles ein gutes Ende findet. Der tschechische Dichter und Politiker Václav Havel (1936-2011) hat eine andere Formulierung gewählt, die einem seelsorglichen Hoffnungsverständnis sehr nahekommt: „Hoffnung ist nicht die Überzeugung, dass etwas gut ausgeht, sondern die Gewissheit, dass etwas Sinn hat, egal wie es ausgeht. "10 Diese Worte beschreiben zielgenau das Wirken helfender Berufe. Sie stehen Menschen zur Seite, die ihre Situation nicht selten als hoffnungslos bezeichnen. In solchen Situationen selbst Hoffnung auszustrahlen, ist wahrlich eine Kunst. Dabei geht es nicht darum, die Probleme klein- oder schönzureden, sondern sich gemeinsam auf die Suche nach dem Sinn zu machen. Und den gibt es nach dem Psychiater Viktor E. Frankl (19051997) in jeglicher Lebenssituation, denn das Leben hat seiner Überzeugung nach sogar einen bedingungslosen Sinn. Dabei wären wir allerdings schlecht beraten, die Corona-Krise vorschnell zu deuten. Nicht wenige tun das, schmieden Verschwörungstheorien, versuchen sie als Ergebnis unseres Handelns einzuordnen oder gar als Strafe Gottes auszumachen. Es geht folgt man Viktor E. Frankl - vielmehr um den je eigenen Lebenssinn, der, unter welchen Umständen auch immer, zu suchen und zu finden ist. Und die Gewissheit, dass sich jeden Tag so ein Sinnanruf für jeden Menschen

8 Vgl. dazu Rosa, Resonanz, 435-453.

9 Vgl. Rosa, Folgen der Coronakrise.

10 Havel, Zitate. 
ganz persönlich finden lässt, ist unter schwierigen Bedingungen außerordentlich hoffnungsvoll, selbst wenn noch nicht greifbar ist, wohin die Krise schließlich führen wird. Viktor E. Frankl hat seine Überlegungen immerhin selbst unter furchtbarsten Bedingungen im Konzentrationslager bestätigt gefunden. Seine schweren und doch hoffnungsstiftenden Erkenntnisse aus dieser Zeit sind in seinem Buch ... trotzdem Ja zum Leben sagen $^{11}$ dargelegt. Dieser Titel ist auch bezeichnend für den Hoffnungsdienst von Seelsorge.

\subsubsection{Liebe: „Es ist was es ist“" (E. Fried)}

Liebe ist ein strapaziertes Wort. Im paulinischen Zusammenhang (1 Kor 13) stehen nicht die Emotionen im Vordergrund. Vielmehr ist eine innere Zustimmung zu einem Menschen oder einer Situation gemeint, selbst wenn es Gründe gibt, die den Menschen oder die Situation unliebsam erscheinen lassen. Paulus schreibt: Liebe „erträgt alles, glaubt alles, hofft alles, hält allem stand" (1 Kor 13,7). Man könnte mit einem anderen Begriff vielleicht sagen: Liebe ist die Kunst, etwas gut sein zu lassen. Der oben bereits zitierte Erich Fried bringt es in seinen sehr eingänglichen Zeilen auf den Punkt: "Es ist was es ist / sagt die Liebe. "12 Dazu gehört letztlich auch die Unverfügbarkeit des Lebens samt seiner Zerbrechlichkeit und Sterblichkeit. Bundestagspräsident Wolfgang Schäuble hat als einer der ganz Wenigen öffentlich auf dieses Thema hingewiesen:

„Wenn ich höre, alles andere habe vor dem Schutz von Leben zurückzutreten, dann muss ich sagen: Das ist in dieser Absolutheit nicht richtig. Wenn es überhaupt einen absoluten Wert in unserem Grundgesetz gibt, dann ist das die Würde des Menschen. "Die Würde des Menschen sei unantastbar. Das schließe aber nicht aus, „dass wir sterben müssen“.13

11 Siehe Frankl, ...trotzdem Ja zum Leben sagen.

12 Fried, Was es ist, 43.

13 Schäuble, zit. nach Hummler, Im Tod ist das Leben, 235. Der Krankenhausseelsorger Georg Hummler kritisiert, dass solche Worte von Seiten der Kirche kaum zu hören waren. Er schreibt weiter: „Die Corona-getriebene existenzielle (Todes-)Angst vieler Menschen bedarf einer öffentlichen religiösen Deutung so dringend wie kaum zuvor. Die Hochleistungsmedizin erfährt den Tod als Entmachtung ihrer Machbarkeitsvorstellungen. Ja, dadurch wird manche medizinische Selbstüberschätzung schwer getroffen. Als Klinikseelsorger habe ich die Not der Ärzte und der Pflegekräfte erlebt, wenn sie dem Diktat der Todesverhinderung um jeden Preis nicht entkommen konnten." (Ebd.). 
Solche Themen anzusprechen, gilt in der Öffentlichkeit schon beinahe als Zumutung. Es aus Liebe zum Leben dennoch zu wagen, ist wesentliche Aufgabe von Seelsorge.

\subsection{Kreative Seelsorge: Möglichkeiten aus-SCHÖPFEN}

Seelsorge ist schließlich dann richtig und notwendig, ja systemrelevant, wenn sie kreativ ist - im wahrsten Sinne des Wortes schöpferisch. Wie das Buch Genesis am Anfang der Heiligen Schrift das Schöpfungsgeschehen als Wirken des Gottesgeistes beschreibt, so arbeitet geistliche Begleitung oder Seelsorge fortwährend an einer schöpferischen Lebensgestaltung mit. Und dazu gehört der Raum zum Atmen.

Seelsorge ist dann kreativ, wenn sie kein spirituelles „Fertiggericht“ anbietet, sondern gemeinsam mit dem Hilfsbedürftigen „kocht“. Die frühere Rede von den Schätzen oder den Heilsmitteln der Kirche suggerierte, dass es da etwas gebe, das man sich geben lassen könne, damit es einem besser gehe. Es wird allerdings zunehmend deutlich, dass die Bedürfnisse immer individueller werden, sodass in der Folge auch die Angebote immer mehr auf die Nachfragenden zugeschnitten werden müssen. In der Praxis Jesu wurde das wiederholt sichtbar in seiner Frage „Was willst du, dass ich dir tue?" (Mk 10,51). Das Entscheidende ist das Erkennen einer Not und das Angebot der Unterstützung. Worum es letztlich geht, wird sich in der Begegnung zeigen.

Was kreative Seelsorge anbieten kann, ist Heilung und Trost. Jesu Zuwendung zu den Hilfsbedürftigen hatte immer etwas mit Heil zu tun nicht zuerst mit dem ewigen Seelenheil des Menschen, sondern zunächst immer mit einem Mehr an Lebensqualität durch tatsächliche Heilung, aber auch durch Vergebung, durch das offene Ansprechen von Verwundungen im Leben und durch die Förderung der Bereitschaft zu einem Neuanfang. All das erlebten Menschen damals als heilsam - und so erleben sie es auch heute. Da kommt der zweite Begriff ins Spiel: Trost. Trost hat nichts mit Vertrösten zu tun. Es gibt ein indogermanisches Wort, deru, das Eiche oder Baum bedeutet. Im englischen Wort tree ist es noch zu finden und dann auch in Ableitungen wie dem englischen trust, auf Deutsch Vertrauen, Treue, Trost. Es geht dabei immer um eine Unterstützung, die stabilisierend ist, eine Hilfe, an die man sich vergleichsweise anlehnen kann wie an einen Baum. Dass der Heilige Geist auch Tröster genannt wird, leitet sich vom griechischen Wort parakletos ab, das so viel bedeutet wie der Herbeigerufene, der zu Hilfe eilt, der Beistand. Seelsorge ist heilsamer Beistand für all diejenigen, die momentan selbst zu wenig Kraft zum Le- 
ben oder auch Kraft zum Helfen haben. Um solchen Beistand leisten zu können, bedarf es einer guten Beziehung - und eines großen Stücks Kreativität.

\section{Sein Unglück ausatmen können}

Seelsorge ist Atemhilfe - für die Kranken und Notleidenden ebenso wie für die Helfenden. Wenn sie bereit ist, sich auf die Regeln des Systems einzulassen, dann ist sie im höchsten Maß systemrelevant. Aber man wird mit Hartmut Rosa den Begriff Systemrelevanz genauer definieren müssen:

„Systemrelevant sollte eben nicht heißen: Was ist relevant für das Aufrechterhalten der Finanzmärkte, sondern was ist relevant und wichtig für das Aufrechterhalten unseres Lebens, für ein gelingendes Leben. "14

\section{Literatur}

Domin, Hilde: Es gibt dich, in: Dies.: Der Baum blüht trotzdem. Gedichte, Frankfurt am Main: S. Fischer ${ }^{2} 1999,56$.

Frankl, Viktor E.: ...trotzdem Ja zum Leben sagen. Ein Psychologe erlebt das Konzentrationslager, München: Penguin ${ }^{3} 2018$.

Fried, Erich: Aufhebung, in: https://www.deutschelyrik.de/aufhebung-1100.html [23.06.2020].

Fried, Erich: Was es ist, in: Ders.: Es ist was es ist. Liebesgedichte - Angstgedichte Zorngedichte, Berlin: Klaus Wagenbach 1999, 43.

Havel, Václav, Zitate, in: https://www.zitate.eu/autor/vaclav-havel-zitate/220290 [23.06.2020].

Hölderlin, Friedrich: Patmos, in: Hölderlin-Gedichte. Herausgegeben und mit Erläuterungen versehen von Jochen Schmidt, Frankfurt am Main: Insel-Verlag 1969 (= insel taschenbuch 781, 1984), 176-183.

Hummler, Georg: Im Tod ist das Leben, in: Christ in der Gegenwart 22 (2020) 235.

Papst Franziskus: Apostolisches Schreiben Evangelii Gaudium, 30, in: http://www. vatican.va/content/francesco/de/apost_exhortations/documents/papa-francesco_e sortazione-ap_20131124_evangelii-gaudium.html\#Eine_unaufschiebbare_kirchli che_Erneuerung [23.06.2020].

14 Rosa, Folgen der Coronakrise. 
Rosa, Hartmut: Folgen der Coronakrise. Was in unserer Gesellschaft wirklich systemrelevant ist, in: https://www.deutschlandfunk.de/folgen-der-coronakrise-wasin-unserer-gesellschaft-wirklich.886.de.html?dram:article_id $=477022$ [07.06.2020].

Rosa, Hartmut: Resonanz. Eine Soziologie der Weltbeziehung, Berlin: Suhrkamp 2016.

Saunders, Cicely, zitiert nach Palliamo, in: https://www.palliamo.de/patienten-undangehoerige/palliative-care/ [23.06.2020].

Weltgesundheitsorganisation (WHO): Definition of Palliative Care 2002, in: https:/ /www.dgpalliativmedizin.de/images/stories/WHO_Definition_2002_Palliative Care_englisch-deutsch.pdf [23.06.2020]. 
